\title{
The admission of patients with pneumonia directly from the Emergency Department to Hospital in the Home
}

\section{Michael Montalto}

\begin{abstract}
\section{Introduction}

Acute respiratory infection is often the commonest reason for patients to be admitted to hospital from the Emergency Department (ED). Hospital in the Home Units (HHUs) deliver acute hospital type care to patients at home. This study sought to review the safety and effectiveness of HHU care for patients with pneumonia referred directly from an ED to a HHU using standard selection criteria.
\end{abstract}

\section{Method}

A retrospective clinical audit of all patients with acute pneumonia referred from the ED to the HHU from March 2000 to March 2002.

\section{Results}

45 episodes in 44 patients were identified. 19 patients were female and the mean age was 53 years. Seven $(15.6 \%)$ patients were residents of nursing homes.

In $23(51.1 \%)$ episodes, patients had presented to ED already taking oral antibiotics for their current condition. In 30 patients, significant co-morbidities were identified.

The mean temperature on presentation was $37.7^{\circ}$ Centigrade. The range of oxygen saturation was $89-100 \%$, with a mean of $95 \%$. Positive chest xray findings were reported in $41(91.1 \%)$ of 45 episodes. In $9(20 \%)$ cases an effusion was also reported.
Length of stay ranged from 1-20 days with a mean of 6.2 days. In all 45 episodes, patients were treated with ceftriaxone. Adjuvant oral therapy was initiated in 28 episodes. Other adjuvant therapy was initiated at admission: nebulised salbutamol (7); oral prednisolone (1).

Intravenous crystalloid fluids were required at home for rehydration in 2 episodes. Antibiotic therapy was altered in two cases. Oral prednisolone was commenced in 4 episodes, nebulised salbutamol commenced in 7 episodes, and fluconazole and frusemide were each commenced in a single episode.

In three cases the primary discharge diagnosis was not related to respiratory infection.

In 5 episodes $(11.1 \%)$, patients made an unplanned telephone call to HHU staff. In 4 (8.9\%) episodes, an unplanned staff visit was required. One patient required a return to hospital to complete her inpatient treatment. Two patients were readmitted within 1 month of discharge from EHHU.

\section{Conclusion}

Patients who present to EDs with respiratory infection requiring intravenous therapy may be safely referred directly into Hospital in the Home care.

\section{Michael Montalto \\ Director \\ Epworth Hospital in the}

Home Unit

Correspondence to:

89 Bridge Rd,

Richmond, Victoria

Australia 3121

Tel: +61 (0)3 94268544

Fax: +61 (0)3 94266856

Email

michaelm@epworth.org.au

Date submitted: $22 / 10 / 02$

Date Accepted: 10/02/03

Prim Care Resp J 2003;

12(1):12-15

\section{Introduction}

Acute respiratory infection is an extremely common presentation to Emergency Departments. In the Australian State of Victoria, acute lobar pneumonia (ICD 10: J181) and lower respiratory tract infections (ICD 10: J22) accounted for 9593 presentations to all Emergency Departments in 2000-2001. ${ }^{1}$ Of these presentations, 7,218 patients were admitted to hospital ( $3 \%$ of all admissions to hospital), where they spent more than 30,000 hospital inpatient bed days. 3,792 of these patients $(55 \%)$ were aged over 65 . Acute respiratory infection was the commonest reason for admission to hospital from Emergency Departments in Victoria for the last two years 1999-2001. The average mortality rate for community acquired pneumonia in hospital is approximately $14 \% .^{2}$ The development of winter bed strategies is mainly targeted at the seasonal influx of respiratory infections, which places a heavy burden on Emergency Departments and hospitals. ${ }^{3}$

Hospital in the Home care refers to the management of acute hospital conditions in the patients' homes, where otherwise inpatient care would be required. This includes the treatment of a wide range of infections and thromboembolic disease. The direct transfer of patients from Emergency Departments to Hospital in the Home has been reported previously. ${ }^{4}$
That work suggested that such transfers can be safe and effective, as long as adequate assessment and vigilant supervision is provided by the Hospital in the Home Unit.

The aim of this study is to examine the safety and effectiveness of direct referral of patients diagnosed with pneumonia in the Emergency Department for treatment in a medically managed Hospital in the Home Unit. It will validate the assessment criteria used to select appropriate patients for such care, and standard Hospital in the Home pneumonia treatment strategies.

\section{Setting}

Epworth Hospital is a private not for profit hospital in Melbourne Australia. It is the largest single campus hospital in Australia with 500 beds. Epworth Emergency is a busy department staffed with Emergency Physicians.

\section{Epworth Hospital in the Home (EHHU)}

Epworth Hospital in the Home is a distinct, fully integrated hospital based unit that accepts patient referrals from the Emergency Department at any time. The HHU assessment criteria for patients with pneumonia are described in Box 1. Suitable patients are formally admitted to Epworth Hospital as inpatients. Nursing staff are employed specifically to 
the unit, and deliver all care, including antibiotic dosing, pathology testing, monitoring of vital signs including oxygen saturation, and recording of clinical information. 24 hour nursing cover specific for HHU is provided. Specifically appointed medical staff (with general practice backgrounds) manage HHU patients. The doctors are responsible for the patients during their stay, and initiate treatment, investigations, and visit and examine each patient at home on a regular basis. They communicate with usual practitioners on discharge. They also provide specific 24 hour cover.

\section{Method}

This study uses a retrospective clinical audit design, using a census of all consecutive patient episodes for the sample population.

Acute lower respiratory tract infection was the subject of this study. Cases where the primary treated condition was an exacerbation of chronic obstructive or inflammatory airways/asthma were excluded from the study population. Where airways disease was a contributing factor to either the condition or its treatment, this was noted in the co-morbidities. Referrals directly into the EHHU without contact with an Emergency Department were also excluded from this study population.

Epworth Hospital in the Home Unit maintains a standard database for all EHHU patient episodes. This database was interrogated to find all episodes of pneumonia and lower respiratory tract infection admitted into EHHU where the referring department was identified as an Emergency Department. Referring departments could be Epworth Emergency Department or any other Emergency Department. All patient episodes occurring between March 2000 and March 2002 were identified. The original medical record was examined for each identified case.

Data were extracted that related to patient characteristics, the baseline severity of the condition at presentation, ${ }^{5}$ investigation and treatment options, and outcomes. Data were extracted from the database and the medical record (Box 2).

\section{Results}

\section{Patient characteristics}

There were 45 patient episodes identified in 44 patients. The average age was 53 years (range 17-92). 25 patients were male, 19 were female. 7 (15.6\%) patients were residents of nursing homes. $25(55.6 \%)$ patients were over 50 years of age.

\section{Prior oral antibiotic use}

In $23(51.1 \%)$ episodes, patients had presented to ED already taking oral antibiotics for their current condition.

\section{Co-morbidities}

In 14 patients, no important co-morbidity relating to the respiratory infection could be identified. In the remaining 30 patients, the co-morbidities were identified and are listed in Table 1. Multiple co-
Box 1: Criteria for assessing suitability for EHHU care in patients referred from ED with pneumonia

Assessment criteria for HHU care: pneumonia

- No acute confusion

- Adequate carer support

- Oxygen saturation $>90 \%$ in room air

- Systolic BP $>90 \mathrm{mmHg}$

- Pulse $<120$ beats per minute

- Respiratory rate $<30$ breaths per minute

- No new left ventricular failure

- Patient consent

Box 2: Data extracted from the database and the medical records

1. Age

2. Sex

3. Significant co-morbidities

4. Oral antibiotic on presentation to ED

5. Temperature in ED (highest of readings in ED)

6. Pulse (highest in ED)

7. Oxygen saturation in room air (lowest in ED)

8. Systolic blood pressure (lowest in ED)

9. Respiratory rate (highest in ED)

10. Chest xray findings

11. Sputum micro result

12. Blood culture result

13. Treatment

14. Change/addition to treatment after initiation

15. Length of stay

16. Unplanned patient telephone call to EHHU, as defined by Australian Council on Healthcare Standards

17. Unplanned staff visit, as defined by Australian Council on Healthcare Standards

18. Unplanned return to hospital, as defined by Australian Council on Healthcare Standards

19. Readmission for related condition within one month

20. Diagnosis at discharge different to diagnosis at entry morbidities were included

Presenting clinical features

The mean temperature was

$37.7^{\circ}$ Centigrade (range $35.4-$ 40.3).

The range of oxygen saturation was $89-100 \%$, with a mean of $95 \%$, however in one patient, supplemental oxygen of $3 \mathrm{~L} / \mathrm{min}$ was maintained.

\section{Table 1. Significant co-morbidities in patient with pneumonia admitted in EHHU}

Co-morbidity number of patients

Asthma/COPD

$8(17.8)$

Dementia

$6(13.3)$

Cancer: current

$5(11.1)$

Smoking

$4(8.9)$

CVA

Other IHD

$4(8.9)$

NIDDM

$3(6.7)$

New AF

$2(4.4)$

Post-partum

$2(4.4)$

Respiratory rate was

recorded in a range from 18-

42 respirations per minute, with a mean of 22 .

Recent surgery

Splenectomy

Alcoholism

Hypopituatrism

The mean pulse rate was 91 , with a range from $65-138$.

ABI with tracheostomy

\section{Presenting diagnostic tests}

Positive chest xray findings were reported in 41 (91.1\%) of 45 episodes. Left lower lobe consolidation was commonest (13 or $28.9 \%$ ), with 9 patients demonstrating right lower lobe consolidation. Bilateral changes were reported in 5 patients. The remaining abnormalities were: right middle lobe 
consolidation (4); right upper lobe consolidation (3); left lingula consolidation (3); left atelectasis (2) and left upper lobe consolidation (1). In 9 (20\%) cases an effusion was also reported.

Only 3 sputum cultures yielded organisms: haemophilus influenzae; aspergillus fumigatus; and branhamella catarrhalis.

In 2 patients, blood cultures grew organisms: a methicillin sensitive staphylocccus aureus and a staphylococcus capitis.

Serology subsequently confirmed one case of legionella, two cases of mycoplasma pneumoniae, and one case of EBV.

\section{Treatment}

The mainstay of treatment for pneumonia in EHHU was intravenous ceftriaxone: in all 45 episodes, patients were treated with ceftriaxone. In one case, intravenous gentamicin was used in conjunction. Simultaneous oral therapy was also initiated: 23 received rothrixomycin; 2 received doxycycline; and 3 received metronidazole. Other adjuvant therapy was initiated at admission: nebulised salbutamol (7 episodes); oral prednisolone (1 episodes).

Intravenous crystalloid fluids were required at home for rehydration in 2 episodes. Antibiotic therapy was altered in two cases, one where ceftriaxone was replaced with cefepime, and in another where all antibiotic therapy was ceased. Oral prednisolone was commenced in 4 episodes, nebulised salbutamol commenced in 7 episodes, and fluconazole and frusemide were each commenced in a single episode.

\section{Discussion}

This study suggests that the assessment criteria for pneumonia used in Epworth HHU may deliver satisfactory outcomes in the acute management of this illness where patients are referred directly from an emergency department. The baseline criteria, in association with further assessment by HHU medical staff, may predict a safe and effective outcome for the treatment of pneumonia directly from Emergency Departments. This is, however, a descriptive study with a small population sample. Caution in interpretation is required, and the constituent features of the EHHU model employed that may result in the outcomes described here need to be carefully considered.

All the patients agreed to enter HIH care after assessment. Refusal by patients assessed as suitable for HIH occurred in one instance, although it is possible that patients expressed reluctance for acute home based care to their attending emergency physician prior to a formal referral to $\mathrm{HIH}$.

In many episodes, interventions were made by medical and nursing staff that may have positively influenced outcomes. These may have occurred during regular visits, or during unplanned interventions. In 17 cases $(37.8 \%)$ studied here, changes to the treatment regime were documented in response to the clinical progress after the patient left the Emergency Department. In more than $20 \%$ of admissions (10), unplanned interventions (telephone calls, callouts or returns to hospital) were experienced. Deterioration in acute respiratory infection may occur rapidly, even where no specific risk factors for poor outcome are identified beforehand. In high risk groups, such as nursing home residents, pneumonia is associated with mortality rates of up to $21 \%{ }^{6}$ The early identification and treatment of new symptoms, in particular the emergence of inflammatory airways disturbance, revision of the initial diagnosis, and the alleviation of anxiety among this group are important benefits of the model of care used in this study.

This supports the establishment of a clinical unit approach, where care is provided in HHU by a small group of HHU medical and nursing staff, who also provide 24 hour 7 day cover for the patients while in HHU. Frequent medical home visits are important in the delivery of these outcomes. Some trials of hospital in the home care have not included this level of medical support, and mortality has been reported in Hospital in the Home arms. ${ }^{7}$ While mortality may be expected in caring for a group of patients with serious respiratory infections, particularly the very elderly, it should be injury, permanent tracheostomy and splenectomy returned with recurrent pneumonia. Both patients were subsequently discharged in good condition. 
ensured that this does not result from decreased quality of care.

Although formal risk scoring was not performed, the characteristics of the patient group in this study are broadly typical of those admitted to hospital (excluding intensive care) for acute pneumonia. All patients were referred for acute care by Emergency Department consultants. Of interest is that half of the patients in the study had failed a trial of oral therapy. Significant co-morbities including nursing home residence, age, chronic airways disease, cancer, ischemic heart disease and others were present in this cohort. However, these conditions do not, in themselves, preclude treatment in a properly organised HHU even though they predict worse outcomes for pneumonia. Four patients did not have radiological changes, but were still diagnosed with acute pneumonia on other clinical criteria. The three patients with final diagnoses other than pneumonia at discharge were included to demonstrate the outcomes of all patients admitted with pneumonia: diagnostic uncertainty is a part of the management of such patients. The assessment of respiratory status in patients with dementia is difficult, but the outcomes demonstrated in this subgroup support the adequacy of the assessment and the management.

The choice of therapy is influenced by HHU.

Ceftriaxone has two major advantages in this setting: it can be used as a single daily dose and its spectrum and effectiveness ensures that treatment failures are minimised. The use of this more potent alternative offers greater security to treating medical staff, in the situation where patients are necessarily monitored less often than they would be in a traditional hospital setting. Rehydration at home should be undertaken with care. The aim of rehydration is supportive, not resuscitative. In this population one litre of crystalloid each day was infused where required, with close clinical and biochemical monitoring.

Hospital in the Home has the ability to respond to the dramatic burden placed on emergency departments and hospitals by acute severe respiratory illness. This includes the treatment of elderly patients resident in nursing homes. Patients will accept properly organised hospital in the home alternatives to traditional hospitalisation. ${ }^{8}$ The relationship between Emergency Departments and Hospital in the Home should be equivalent to that between ED and any other acute hospital unit. Emergency Departments should broadly understand HHU assessment criteria and offer HHU a role in treating acutely unwell patients. HHUs should ensure that the service is of high quality, access to the service is always available and timely in its response. In this way, a realistic basis for the treatment of pneumonia and a range of other acute conditions referred directly from Emergency

Departments can be achieved. Economic data has not been included, as this is primarily a clinical paper, and a cost study was beyond the scope of the study. The level of clinical support and medication used would suggest that recurrent costs of patient management in this manner may not be dramatically less than usual hospital care. The ability for HIH to rapidly build new beds for overburdened hospitals at relatively low capital cost is an important feature of the economic advantage of $\mathrm{HIH}$.

Similar criteria of assessment for direct entry into HHU can be created for a number of conditions that frequently present to the Emergency Department, including: cellulitis; DVT; pyelonephritis; epididymoorchitis and tonsillitis. If the use of validated screening criteria is implemented, and HHUs are adequately staffed, then HHU referral should be used as a routine ward destination for these patients. This avoids using HHUs only in times of overflow. By referring all appropriate patients to HHU when they present, a significant impact on hospital bed utilisation can be made. This requires co-operation between Emergency Departments and adequately staffed HHUs, and offers an exciting development in the future of acute hospital care.

\section{References}

1. Hospital Admission Risk Program Background Paper Emergency Demand Co-ordination Group Victorian Department of Human Services March 2002 Melbourne.

2. Johnston P, Irving L, Turnidge J 'Community acquired pneumonia' Med J Aust 2002; 176: 341-7 3. Hanratty B, Robinson M 'Coping with winter bed crises' BMJ 1999; 319:1511-2.

4. Montalto M 'An audit of patients admitted for home intravenous therapy directly from the emergency department' International Journal of Clinical Practice 1997: 51(7): 433-437.

5. Fine M, Smith M, Carson C et al Prognosis and outcomes of patients with community acquired pneumonia. A meta analysis. JAMA 1996; 275: 134 141.

6. Naughton B, Mylotte J, Tayara A 'Outcome of nursing-home-acquired pneumonia' $J$ Am Geriatric Soc 2000; 48:1292.

7. L Davies, M Wilkinson, S Bonner, P M A Calverley, R M Angus "Hospital at home" versus hospital care in patients with exacerbations of chronic obstructive pulmonary disease: prospective randomised controlled trial BMJ 2000; 321: 1265-1268.

8. Montalto M 'Patients' and carers' satisfaction with Hospital in the Home care' Int J Qual Health Care 1996; 8(3):243-254. 